\title{
A novel method for vaginal cylinder treatment planning: a seamless transition to 3D brachytherapy
}

\author{
Harish K. Malhotra, PhD, Vincent Wu, PhD, Zhou Wang, PhD, Sachin Patil, MD \\ Department of Radiation Medicine, Roswell Park Cancer Institute, Buffalo, USA
}

\begin{abstract}
Purpose: Standard treatment plan libraries are often used to ensure a quick turn-around time for vaginal cylinder treatments. Recently there is increasing interest in transitioning from conventional 2D radiograph based brachytherapy to 3D image based brachytherapy, which has resulted in a substantial increase in treatment planning time and decrease in patient through-put. We describe a novel technique that significantly reduces the treatment planning time for CT-based vaginal cylinder brachytherapy.

Material and methods: Oncentra MasterPlan TPS allows multiple sets of data points to be classified as applicator points which has been harnessed in this method. The method relies on two hard anchor points: the first dwell position in a catheter and an applicator configuration specific dwell position as the plan origin and a soft anchor point beyond the last active dwell position to define the axis of the catheter. The spatial location of various data points on the applicator's surface and at $5 \mathrm{~mm}$ depth are stored in an Excel file that can easily be transferred into a patient CT data set using window operations and then used for treatment planning. The remainder of the treatment planning process remains unaffected.

Results: The treatment plans generated on the Oncentra MasterPlan TPS using this novel method yielded results comparable to those generated on the Plato TPS using a standard treatment plan library in terms of treatment times, dwell weights and dwell times for a given optimization method and normalization points. Less than $2 \%$ difference was noticed between the treatment times generated between both systems. Using the above method, the entire planning process, including CT importing, catheter reconstruction, multiple data point definition, optimization and dose prescription, can be completed in $\sim 5-10$ minutes.

Conclusion: The proposed method allows a smooth and efficient transition to 3D CT based vaginal cylinder brachytherapy planning.
\end{abstract}

Key words: vaginal brachytherapy, high-dose-rate brachytherapy.

\section{Purpose}

High Dose Rate (HDR) brachytherapy using vaginal cylinders is increasingly being used for delivering radiation dosage in the postoperative setting for several gynecologic malignancies [1-8]. For endometrial cancer, hysterectomy followed by vaginal brachytherapy (VBT) offers similar local control with less toxicity as compared to adjuvant external beam radiation therapy (EBRT) to the pelvis [3,4]. When compared to interstitial brachytherapy or other applicator devices such as tandem and ovoids, VBT using vaginal cylinders offers additional advantages in its ease of placement, minimal discomfort to the patient and simplicity of treatment planning. Overall shorter treatment time, both total number of treatments and time spent per session, as well as the relatively lower associated cost have further led to its being preferred modality for vaginal cylinder brachytherapy.

In $2 \mathrm{D}$ radiograph based brachytherapy, simple treatment planning has traditionally been performed using standard orthogonal films to construct a treatment plan usually with- in a matter of minutes. Due to the fact that the applicator usually has only a single straight catheter and discrete rigid cylinder diameters, many centers create a standard plan library in which the entire applicator geometry along with the necessary data points is stored on the treatment planning system (TPS) itself for a quick recall and subsequent use as a patient plan without incorporating any other information regarding organs at risk (OARs) or other patient related anatomical reference points during treatment planning. X-ray films were usually used to verify the positioning of the applicator in a patient and a quick quality assurance to verify cylinder diameter. This made the entire process extremely efficient.

Recently there has been increasing interest to move to 3D image based brachytherapy to allow for a more accurate volumetric assessment of the radiation dose delivered to the target (vaginal cuff) and nearby critical structures (such as bladder and rectum) $[1,9,10]$. The incorporation of 3D CT data into the treatment planning process, however, comes 
at a potential cost of increased treatment planning time. This added time is related to having to identify and mark clinically relevant points for dose prescription and evaluation within the CT data set. As per the American Brachytherapy Society (ABS) reporting guidelines for endometrial cancers, dose points need to be placed along the applicator surface as well as at a depth of $5 \mathrm{~mm}$ [9]. Since there is not a guaranteed benefit in plan quality or clinical outcome with the use of 3D based plans as compared to 2D based plans, there has been a reluctance to move to 3D brachytherapy for vaginal cylinders.

A novel solution to this, which allows for the transition from $2 \mathrm{D}$ to $3 \mathrm{D}$ brachytherapy, but keeps planning time down, takes an advantage of the fact that radiation dose prescription as well as treatment plan reporting criteria are linked to the applicator rather than a patient specific anatomical point (viz. cylinder surface or at a depth of $5 \mathrm{~mm}$ into tissue). This allows for the creation of a treatment plan library in which the spatial coordinates for a set of dose points can be stored according to cylinder diameter, quickly recalled and overlaid on the patient's actual CT data set in a timely manner for dose prescription and plan evaluation. This process has worked with great success to keep planning times to a minimum.

Over the last several years, new TPSs like Oncentra MasterPlan (ver. 3.3 SP3 from Nucletron B.V., Waardgelder 1, 3905TH Veenendaal, PO Box 930, 3900 AX Veenendaal, The Netherlands) have been introduced to facilitate the transition from 2D to 3D HDR brachytherapy. Unfortunately, the Oncentra MasterPlan TPS does not yet have an option to create a standard treatment plan library as outlined above. Each individual plan thus has to be developed from scratch, leading to substantially longer planning times.

We describe a simple, quick and efficient method for converting a pre-existing treatment plan library from Plato ver. 14.3.5 TPS (a precursor) to the Oncentra MasterPlan system (ver. $3.3 \mathrm{SP} 3$ ) and enhancing it further, so that it can even be overlaid on the patients CT data set and further customized to each particular patient, if needed. These plans contain all the necessary dose points for dose prescription, as well as plan evaluation. We expect a significant reduction in planning time as well as potential reductions in planning error and meaningful improvements in patient throughput. Moreover, the system makes it feasible to use volumetric indices for plan evaluation using the base 3D CT data set.

\section{Material and methods}

Every CT scan has its own coordinate system called patient coordinate system that employs a DICOM origin, the location of which depends on the patient positioning during CT scanning. Because of its variability from patient to patient, this coordinate system is not very useful for the incorporation of standard treatment plans. Oncentra MasterPlan also supports applicator coordinate system which utilizes a user defined origin. This user-defined origin is associated with the treatment applicator and can be easily changed by the treatment planner. Since the radiation delivery in these patients is associated with the dose delivery to the applicator cylinder surface or $5 \mathrm{~mm}$ from it, the applicator coordinate system is more useful for the creation of standard treatment plan library. In addition, it can have multiple datasets associated to it allowing a creation of two separate sets of data points at the applicator surface and at a depth of $5 \mathrm{~mm}$ satisfying the ABS reporting guidelines for the endometrial cancers [9]. Our method of creating a standard treatment plan library utilizes this coordinate system within Oncentra MasterPlan. The entire treatment plan library is stored in a separate Microsoft Excel file, a vendor approved software which could be installed on Oncentra MasterPlan TPS. Using the standard "copy" and "paste" windows operations, it is possible to import and overlay a treatment plan from this standard plan library in a patient 3D CT dataset. The imported data set then becomes a part of the patient treatment plan and can be used for treatment plan prescription as well as evaluation.

In doing so, a library of treatment plans was created and stored on the Oncentra Master Plan TPS. Each plan was custom-made to fit given clinical scenario based on various cylinder sizes $(2.0,2.5,3.0,3.5 \mathrm{~cm}$ diameter) and loading patterns (the number and location of active dwell positions). The treatment plan that matches the implant/treatment geometry could then be selected when the patient arrives for treatment with minor modifications for dose calculation purposes as needed.

\section{Creation and importation of a plan library from Plato TPS}

The physicians at our institution are comfortable with the treatment plans that were previously created on Plato TPS (ver. 14.2) and in clinical use since more than a decade [6]. Thus, it was important to create standard treatment plans on Oncentra MasterPlan TPS with the same loading pattern and dose points defined on the applicator surface and at $5 \mathrm{~mm}$. On Plato TPS, applicator points were defined on the surface of the cylinder while dose points were defined at $5 \mathrm{~mm}$ from the cylinder surface. Grouping of the points in this way was needed for operational purposes as Plato TPS did not support sub-classification of these points. Both points included the curve on the cylinder towards the tip of the applicator where it touches the patient's vaginal cuff. Since this information was already available in a digital format on the Plato TPS, it was considered easier to extract and convert in a format suitable for Oncentra MasterPlan TPS. A dummy patient on Plato TPS was used to pull a standard vaginal plan from its plan library. This brought the catheter reconstruction information, dwell stepsize used, the applicator points and dose point coordinates from the standard plan into the new treatment plan. Using the "jot" program on the Plato TPS, the entire treatment plan was digitally captured in an ASCII format and saved. All of the above points at applicator surface and at $5 \mathrm{~mm}$ distance from it were correlated with the catheter reconstruction points in relation to the current origin in the plan which was recorded for proposed Oncentra MasterPlan TPS plan library. The treatment plan origin coincides with one of the active dwell position in the plan and forms the second fixed anchor point to be used during subsequent patient treatment planning on Oncentra MasterPlan TPS. The process was re- 
peated for all of the stored treatment plans on the Plato TPS for various cylinder diameters and active dwell position configurations. These files were then remotely copied over to the windows environment using standard ftp commands and subsequently imported in an Excel file.

\section{Creation of a plan library in Excel}

To conform to Oncentra MasterPlan TPS coordinates system, the $y$ and $\mathrm{z}$ coordinates coming from Plato TPS in the above Excel file were transposed. Additional columns were added to each point as per the Oncentra MasterPlan TPS requirement which include "Coord. System", Act. Dose (cGy), Act. Dose (\%), Normalization, Norm. Dose, Optimization, Pt. Rel. Dose and Opt. Weight. It was important to make sure that the value under the Coord. System is set to "Applicator" to ensure that these points are interpreted correctly by Oncentra MasterPlan TPS. Act. Dose (\%), Norm. Dose (\%), Opt. Rel. Dose, Opt. Weight columns should all have 100 as the entry as these are the default values used by Oncentra MasterPlan TPS. Actual Dose (cGy) column could contain the most common dose prescription value in use in the clinic. Normalization and optimization columns could be set to "yes" or "no" on a data set (surface or at $5 \mathrm{~mm}$ ) depending upon the user's preference. The actual dose prescription value and the choice of dose points for prescription and optimization (surface or at $5 \mathrm{~mm}$ depth from the applicator) can be changed on the fly in Oncentra MasterPlan TPS allowing for customization of the treatment plans at the time of planning. It is important that each row and column should have the data to ensure windows paste command is honored by Oncentra MasterPlan TPS.

The process was repeated for all the cylinder diameters $(2.0,2.5,3.0$ and $3.5 \mathrm{~cm})$ and different number of dwell positions being used as part of proposed standard plan library. Once created and verified independently, the Excel file was password protected for any inadvertent changes. This Excel file, when used in conjunction with patients CT scan in a manner described below, can help in reproducing the standard treatment plan on to actual patient CT slices and provide the necessary points for dose prescription and evaluation to conform to MD prescription and reporting standards. This completes the base work needed to port the standard treatment plan library from Plato TPS to Oncentra MasterPlan TPS.

\section{Recalling a plan from standard library}

This new method relies on definition of three anchor points, two of which are fixed in nature while one is visual and useful to define the length of the active catheter. The first fixed anchor point is the location of first dwell position from the tip of the applicator. We still use the existing stainless steel vaginal cylinder applicators, where it is impossible to view the $\mathrm{X}$-ray markers position in the $\mathrm{CT}$ data set and hence alternative imaging methods were needed to properly determine the first dwell position in a catheter. We acquired orthogonal radiographic films for all the cylinder diameters after setting them in a shape as if they are about to be implanted in a patient. A $5 \mathrm{~mm}$ bolus was wrapped around the superior end of the applicator followed by a thin copper wire, so that points at $5 \mathrm{~mm}$ could be seen easily on the images. We also placed standard X-ray markers in the applicator and determined the location of the first dwell point from the external tip end of the applicator. We felt it was necessary to verify this for quality assurance and documentation purposes. In our case, this distance was found to be $6.5 \mathrm{~mm}$ (average, range $6.25-6.75 \mathrm{~mm}$ ). This location served as the first fixed anchor point. For clinics which have CT/MR compatible vaginal applicators, they can view the X-ray marker in the CT scan itself and can use that as the first anchor point.

When a patient comes for vaginal brachytherapy, a CT scan with the applicator in treatment position is obtained and exported to Oncentra MasterPlan TPS. These images are then imported into its database. The brachytherapy planning option within Oncentra TPS is selected and the transverse, sagittal and coronal views are pulled in ECS (Extra Coordinate System). The applicator axis is oriented along the superior-inferior axis of the ECS and a point corresponding to first dwell position is defined $6.5 \mathrm{~mm}$ from the tip of the applicator bisecting the applicator longitudinally. This is our first hard anchor point. The second (soft) anchor point is defined $2-3 \mathrm{~cm}$ below the inferior most active dwell position to be used for the patient. This point defines the maximum catheter length and maximum active position which could be used during subsequent treatment planning. Its actual longitudinal position on the applicator is not important as long as it is centered along the horizontal (short axis of the catheter) and allows enough space for the desired number of active dwell positions. Now the catheter definition option is used and a catheter is defined from the first hard anchor point to the second soft anchor point. The required active dwell positions are activated and the ECS coordinate system is moved to its third anchor point, the point at which the original Plato TPS plan had an origin (the second fixed anchor point). An applicator data point set is created and named as "surface" and the respective points defined on the surface of the applicator are copied and pasted from the Excel work sheet created earlier. Upon importing into Oncentra MasterPlan TPS, their positions are seen on the ECS views and their spatial locations are verified. Another applicator data set is created and named appropriately to show that it contains points at $5 \mathrm{~mm}$ from the applicator. Depending on physician's prescription or protocol requirement [11], either of the data set ("surface" or " $5 \mathrm{~mm}^{\prime \prime}$ ) could be used for treatment plan optimization, dose prescription and normalization. The treatment plan can now be evaluated, approved, printed and exported to the treatment unit in a standard manner. The treatment plan reporting satisfies the ABS reporting guidelines for highdose-rate brachytherapy for carcinoma of the endometrium requirements [9].

\section{Results}

Tables 1, 2, 3 and 4 show the coordinates of the points on the applicator surface and at a distance of $5 \mathrm{~mm}$ for 2.0, $2.5,3.0$ and $3.5 \mathrm{~cm}$ cylinder diameter, respectively. The location of the dwell position coincident with the origin of the treatment plan is also included. The table is categorized 
Table 1. Standard treatment plan library with first 7 active dwell positions for $2.0 \mathrm{~cm}$ cylinder diameters for stainless steel vaginal cylinder applicator (Nucletron). Included are coordinates of the points on the applicator surface and at a depth of $5 \mathrm{~mm}$ from the applicator surface; also included is the location of the dwell position coincident with the origin of the treatment plan. It is important to know that in this table the dose values shown at cylinder surface and at $5 \mathrm{~mm}$ from it are for representative purposes only. Their actual values will change based on the dose-prescription, normalization as well as optimization selected by the user on the fly

\begin{tabular}{|c|c|c|c|c|c|c|c|c|c|c|c|}
\hline \multicolumn{12}{|c|}{ Origin $=3^{\text {rd }}$ dwell position } \\
\hline \multicolumn{12}{|c|}{ Applicator pts - surface } \\
\hline Name & $x[\mathrm{~mm}]$ & $y[\mathrm{~mm}]$ & $\mathrm{z}[\mathrm{mm}]$ & $\begin{array}{l}\text { Coord. } \\
\text { system }\end{array}$ & $\begin{array}{l}\text { Act. dose } \\
{[c G y]}\end{array}$ & $\begin{array}{l}\text { Act. dose } \\
(\%)\end{array}$ & $\begin{array}{c}\text { Normali- } \\
\text { zation }\end{array}$ & $\begin{array}{c}\text { Norm. } \\
\text { dose }(\%)\end{array}$ & $\begin{array}{l}\text { Optimi- } \\
\text { zation }\end{array}$ & $\begin{array}{l}\text { Opt. rel. } \\
\text { dose }\end{array}$ & $\begin{array}{c}\text { Opt. } \\
\text { weight }\end{array}$ \\
\hline $\mathrm{A} 1$ & 0.0 & 16.5 & 0 & Applicator & 1000 & 100 & no & 100 & no & 100 & 100 \\
\hline$A 2$ & 3.5 & 15.8 & 0 & Applicator & 1000 & 100 & no & 100 & no & 100 & 100 \\
\hline A3 & 6.2 & 14.0 & 0 & Applicator & 1000 & 100 & no & 100 & no & 100 & 100 \\
\hline A4 & 8.2 & 11.5 & 0 & Applicator & 1000 & 100 & no & 100 & no & 100 & 100 \\
\hline A5 & 9.2 & 9.2 & 0 & Applicator & 1000 & 100 & no & 100 & no & 100 & 100 \\
\hline A6 & 10.0 & 5.2 & 0 & Applicator & 1000 & 100 & no & 100 & no & 100 & 100 \\
\hline A7 & 10.0 & 0.0 & 0 & Applicator & 1000 & 100 & no & 100 & no & 100 & 100 \\
\hline A8 & 10.0 & -5.0 & 0 & Applicator & 1000 & 100 & no & 100 & no & 100 & 100 \\
\hline A9 & 10.0 & -15.0 & 0 & Applicator & 1000 & 100 & no & 100 & no & 100 & 100 \\
\hline A10 & 10.0 & -10.0 & 0 & Applicator & 1000 & 100 & no & 100 & no & 100 & 100 \\
\hline A11 & 10.0 & -20.0 & 0 & Applicator & 1000 & 100 & no & 100 & no & 100 & 100 \\
\hline \multicolumn{12}{|c|}{ Applicator pts - $5 \mathrm{~mm}$ from applicator surface } \\
\hline Name & $x[\mathrm{~mm}]$ & $y[\mathrm{~mm}]$ & $z[\mathrm{~mm}]$ & $\begin{array}{l}\text { Coord. } \\
\text { system }\end{array}$ & $\begin{array}{l}\text { Act. dose } \\
{[\mathrm{cGy}]}\end{array}$ & $\begin{array}{c}\text { Act. dose } \\
(\%)\end{array}$ & $\begin{array}{c}\text { Normali- } \\
\text { zation }\end{array}$ & $\begin{array}{c}\text { Norm. } \\
\text { dose (\%) }\end{array}$ & $\begin{array}{l}\text { Optimi- } \\
\text { zation }\end{array}$ & $\begin{array}{l}\text { Opt. rel. } \\
\text { dose }\end{array}$ & $\begin{array}{c}\text { Opt. } \\
\text { weight }\end{array}$ \\
\hline A12 & 0.0 & 21.5 & 0 & Applicator & 700 & 100 & yes & 100 & yes & 100 & 100 \\
\hline A13 & 3.0 & 20.5 & 0 & Applicator & 700 & 100 & yes & 100 & yes & 100 & 100 \\
\hline A14 & 5.5 & 19.3 & 0 & Applicator & 700 & 100 & yes & 100 & yes & 100 & 100 \\
\hline A15 & 8.1 & 18.0 & 0 & Applicator & 700 & 100 & yes & 100 & yes & 100 & 100 \\
\hline A16 & 10.0 & 16.5 & 0 & Applicator & 700 & 100 & yes & 100 & yes & 100 & 100 \\
\hline A17 & 12.0 & 14.0 & 0 & Applicator & 700 & 100 & yes & 100 & yes & 100 & 100 \\
\hline A18 & 13.5 & 11.5 & 0 & Applicator & 700 & 100 & yes & 100 & yes & 100 & 100 \\
\hline A19 & 14.8 & 8.5 & 0 & Applicator & 700 & 100 & yes & 100 & yes & 100 & 100 \\
\hline $\mathrm{A} 20$ & 15.0 & 5.0 & 0 & Applicator & 700 & 100 & yes & 100 & yes & 100 & 100 \\
\hline A21 & 15.0 & 0.0 & 0 & Applicator & 700 & 100 & yes & 100 & yes & 100 & 100 \\
\hline$A 22$ & 15.0 & -5.0 & 0 & Applicator & 700 & 100 & yes & 100 & yes & 100 & 100 \\
\hline A23 & 15.0 & -10.0 & 0 & Applicator & 700 & 100 & yes & 100 & yes & 100 & 100 \\
\hline$A 24$ & 15.0 & -15.0 & 0 & Applicator & 700 & 100 & yes & 100 & yes & 100 & 100 \\
\hline A25 & 15.0 & -20.0 & 0 & Applicator & 700 & 100 & yes & 100 & yes & 100 & 100 \\
\hline
\end{tabular}

based on the applicator diameter and number of active dwell positions used in a treatment plan. This table uses the first 7 active dwell positions from the tip of the applicator with a source step size of $5 \mathrm{~mm}$. For clarity in publication, this single worksheet from the Microsoft Excel has been broken down into 4 separate tables. Similar tables were generated for different number of active dwell positions in use in the department. In this table, the most important parameters are the $x, y, z$ coordinates of various points and their classification as "applicator points" as remaining parameters will change based on the dose-prescription, normalization and optimization selected by the user at the time of treat- ment planning. However, having all the items together makes it easy for the Oncentra MasterPlan to accept them using windows standard "copy" and "paste" commands. Figure 1 shows a sample treatment plan from our plan library that was generated using standard dosimetry techniques on the Oncentra MasterPlan TPS and then superimposed on a CT scan of a patient who was treated with a $3 \mathrm{~cm}$ vaginal cylinder. The treatment plan consists of a single catheter with first 7 active dwell positions with a step size of $5 \mathrm{~mm}$. Also shown are the points defined on the applicator surface and at a depth of $5 \mathrm{~mm}$. The congruence of these points in relation to applicator can be easily seen as well. 
Table 2. Standard treatment plan library with first 7 active dwell positions for $2.5 \mathrm{~cm}$ cylinder diameters for stainless steel vaginal cylinder applicator (Nucletron). Included are coordinates of the points on the applicator surface and at a distance of $5 \mathrm{~mm}$; also included is the location of the dwell position coincident with the origin of the treatment plan. It is important to know that in this table the dose values shown at cylinder surface and at $5 \mathrm{~mm}$ from it are for representative purposes only. Their actual values will change based on the dose-prescription, normalization as well as optimization selected by the user on the fly

\begin{tabular}{|c|c|c|c|c|c|c|c|c|c|c|c|}
\hline \multicolumn{12}{|c|}{ Origin $=4^{\text {th }}$ dwell position } \\
\hline \multicolumn{12}{|c|}{ Applicator pts - surface } \\
\hline Name & $x[\mathrm{~mm}]$ & $y[\mathrm{~mm}]$ & $z[\mathrm{~mm}]$ & $\begin{array}{l}\text { Coord. } \\
\text { system }\end{array}$ & $\begin{array}{l}\text { Act. dose } \\
\text { [cGy] }\end{array}$ & $\begin{array}{l}\text { Act. dose } \\
\text { (\%) }\end{array}$ & $\begin{array}{c}\text { Normali- } \\
\text { zation }\end{array}$ & $\begin{array}{c}\text { Norm. } \\
\text { dose (\%) }\end{array}$ & $\begin{array}{l}\text { Optimi- } \\
\text { zation }\end{array}$ & $\begin{array}{c}\text { Opt. rel. } \\
\text { dose }\end{array}$ & $\begin{array}{c}\text { Opt. } \\
\text { weight }\end{array}$ \\
\hline $\mathrm{A} 1$ & 0.0 & 21.5 & 0 & Applicator & 1000 & 100 & no & 100 & no & 100 & 100 \\
\hline $\mathrm{A} 2$ & 5.0 & 20.0 & 0 & Applicator & 1000 & 100 & no & 100 & no & 100 & 100 \\
\hline A3 & 9.3 & 16.5 & 0 & Applicator & 1000 & 100 & no & 100 & no & 100 & 100 \\
\hline A4 & 11.8 & 11.5 & 0 & Applicator & 1000 & 100 & no & 100 & no & 100 & 100 \\
\hline A5 & 12.5 & 5.0 & 0 & Applicator & 1000 & 100 & no & 100 & no & 100 & 100 \\
\hline A6 & 12.5 & 0 & 0 & Applicator & 1000 & 100 & no & 100 & no & 100 & 100 \\
\hline A7 & 12.5 & -5.0 & 0 & Applicator & 1000 & 100 & no & 100 & no & 100 & 100 \\
\hline A8 & 12.5 & -10.0 & 0 & Applicator & 1000 & 100 & no & 100 & no & 100 & 100 \\
\hline A9 & 12.5 & -15.0 & 0 & Applicator & 1000 & 100 & no & 100 & no & 100 & 100 \\
\hline \multicolumn{12}{|c|}{ Applicator pts $-5 \mathrm{~mm}$ from applicator surface } \\
\hline Name & $x[\mathrm{~mm}]$ & $y[\mathrm{~mm}]$ & $z[\mathrm{~mm}]$ & $\begin{array}{l}\text { Coord. } \\
\text { system }\end{array}$ & $\begin{array}{l}\text { Act. dose } \\
\text { [cGy] }\end{array}$ & $\begin{array}{l}\text { Act. dose } \\
\text { (\%) }\end{array}$ & $\begin{array}{c}\text { Normali- } \\
\text { zation }\end{array}$ & $\begin{array}{c}\text { Norm. } \\
\text { dose (\%) }\end{array}$ & $\begin{array}{l}\text { Optimi- } \\
\text { zation }\end{array}$ & $\begin{array}{l}\text { Opt. rel. } \\
\text { dose }\end{array}$ & $\begin{array}{c}\text { Opt. } \\
\text { weight }\end{array}$ \\
\hline $\mathrm{A} 10$ & 0.0 & 26.5 & 0 & Applicator & 700 & 100 & yes & 100 & yes & 100 & 100 \\
\hline A11 & 4.5 & 25.5 & 0 & Applicator & 700 & 100 & yes & 100 & yes & 100 & 100 \\
\hline $\mathrm{A} 12$ & 9.6 & 22.5 & 0 & Applicator & 700 & 100 & yes & 100 & yes & 100 & 100 \\
\hline $\mathrm{A} 13$ & 12.5 & 20.0 & 0 & Applicator & 700 & 100 & yes & 100 & yes & 100 & 100 \\
\hline A14 & 15.5 & 16.5 & 0 & Applicator & 700 & 100 & yes & 100 & yes & 100 & 100 \\
\hline A15 & 16.4 & 13.5 & 0 & Applicator & 700 & 100 & yes & 100 & yes & 100 & 100 \\
\hline A16 & 17.0 & 10.0 & 0 & Applicator & 700 & 100 & yes & 100 & yes & 100 & 100 \\
\hline A17 & 17.5 & 5.0 & 0 & Applicator & 700 & 100 & yes & 100 & yes & 100 & 100 \\
\hline $\mathrm{A} 18$ & 17.5 & 0.0 & 0 & Applicator & 700 & 100 & yes & 100 & yes & 100 & 100 \\
\hline A19 & 17.5 & -5.0 & 0 & Applicator & 700 & 100 & yes & 100 & yes & 100 & 100 \\
\hline $\mathrm{A} 20$ & 17.5 & -10.0 & 0 & Applicator & 700 & 100 & yes & 100 & yes & 100 & 100 \\
\hline A21 & 17.5 & -15.0 & 0 & Applicator & 700 & 100 & yes & 100 & yes & 100 & 100 \\
\hline
\end{tabular}

The treatment plans generated on the Oncentra MasterPlan TPS using this novel method yielded results comparable to those generated on the Plato TPS, using a standard treatment plan library in terms of treatment times, dwell weights and dwell times for a given optimization method and normalization points. A maximum of $1.4 \%$ difference was noticed between the treatment times generated among both the systems. It is important to remember that in Plato TPS the standard plan library feature has both the applicator reconstruction and definition of applicator and dose-points as a single entity. Such a rigid arrangement provides consistent results in terms of treatment times per plan once source strength has been corrected for decay. In our method on Oncentra MasterPlan TPS, definition of catheter reconstruction and applicator points are two independent events. They are combined together using user defined origin which can only be placed by using mouse. Despite maximum zooming, a slight difference between ideal and actual plan is possible due to mouse placement errors which results in slightly different results on case by case basis and explains the slight difference seen between Plato and Oncentra MasterPlan TPS results. To study the exact magnitude of these differences, 10 plans each for 2.5, 3.0 and $3.5 \mathrm{~cm}$ cylinder diameter were studied (we haven't yet treated a $2.0 \mathrm{~cm}$ cylinder diameter patient and hence do have a CT data set of a patient).

The treatment times generated for each case were normalized to deliver $10 \mathrm{~Gy}$ to the vaginal surface for a $10 \mathrm{Ci}$ Ir-192 source. The respective, mean time in seconds and the standard deviation $( \pm \mathrm{SD}(\%))$ were found to be $202.4 \mathrm{sec}$ $\pm 0.5 \%, 262.2 \mathrm{sec} \pm 0.7 \%$ and $320.7 \mathrm{sec} \pm 0.8 \%$. These results demonstrate that the differences between different planners were very small.

Three different planners were asked to do a treatment plan with $2.5,3.0$ and $3.5 \mathrm{~cm}$ cylinder with conventional 
Table 3. Standard treatment plan library with first 7 active dwell positions for $3.0 \mathrm{~cm}$ cylinder diameters for stainless steel vaginal cylinder applicator (Nucletron). Included are coordinates of the points on the applicator surface and at a distance of $5 \mathrm{~mm}$; also included is the location of the dwell position coincident with the origin of the treatment plan. It is important to know that in this table the dose values shown at cylinder surface and at $5 \mathrm{~mm}$ from it are for representative purposes only. Their actual values will change based on the dose-prescription, normalization as well as optimization selected by the user on the fly

\begin{tabular}{|c|c|c|c|c|c|c|c|c|c|c|c|}
\hline \multicolumn{12}{|c|}{ Origin $=3^{\text {rd }}$ dwell position } \\
\hline \multicolumn{12}{|c|}{ Applicator pts - surface } \\
\hline Name & $x[\mathrm{~mm}]$ & $y[\mathrm{~mm}]$ & $z[\mathrm{~mm}]$ & $\begin{array}{l}\text { Coord. } \\
\text { system }\end{array}$ & $\begin{array}{c}\text { Act. dose } \\
\text { [cGy] }\end{array}$ & $\begin{array}{l}\text { Act. dose } \\
(\%)\end{array}$ & $\begin{array}{c}\text { Normali- } \\
\text { zation }\end{array}$ & $\begin{array}{c}\text { Norm. } \\
\text { dose }(\%)\end{array}$ & $\begin{array}{l}\text { Optimi- } \\
\text { zation }\end{array}$ & $\begin{array}{l}\text { Opt. rel. } \\
\text { dose }\end{array}$ & $\begin{array}{c}\text { Opt. } \\
\text { weight }\end{array}$ \\
\hline $\mathrm{A} 1$ & 0.0 & 16.5 & 0 & Applicator & 1000 & 100 & no & 100 & no & 100 & 100 \\
\hline $\mathrm{A} 2$ & 5.7 & 15.0 & 0 & Applicator & 1000 & 100 & no & 100 & no & 100 & 100 \\
\hline $\mathrm{A} 3$ & 10.3 & 11.0 & 0 & Applicator & 1000 & 100 & no & 100 & no & 100 & 100 \\
\hline A4 & 12.7 & 7.7 & 0 & Applicator & 1000 & 100 & no & 100 & no & 100 & 100 \\
\hline A5 & 14.0 & 4.7 & 0 & Applicator & 1000 & 100 & no & 100 & no & 100 & 100 \\
\hline A6 & 15.0 & 0.0 & 0 & Applicator & 1000 & 100 & no & 100 & no & 100 & 100 \\
\hline A7 & 15.0 & -10.0 & 0 & Applicator & 1000 & 100 & no & 100 & no & 100 & 100 \\
\hline A8 & 15.0 & -20.0 & 0 & Applicator & 1000 & 100 & no & 100 & no & 100 & 100 \\
\hline \multicolumn{12}{|c|}{ Applicator pts - $5 \mathrm{~mm}$ from applicator surface } \\
\hline Name & $x[\mathrm{~mm}]$ & $y[\mathrm{~mm}]$ & $z[\mathrm{~mm}]$ & $\begin{array}{c}\text { Coord. } \\
\text { system }\end{array}$ & $\begin{array}{c}\text { Act. dose } \\
\text { [cGy] }\end{array}$ & $\begin{array}{c}\text { Act. dose } \\
(\%)\end{array}$ & $\begin{array}{c}\text { Normali- } \\
\text { zation }\end{array}$ & $\begin{array}{c}\text { Norm. } \\
\text { dose }(\%)\end{array}$ & $\begin{array}{c}\text { Optimi- } \\
\text { zation }\end{array}$ & $\begin{array}{l}\text { Opt. rel. } \\
\text { dose }\end{array}$ & $\begin{array}{c}\text { Opt. } \\
\text { weight }\end{array}$ \\
\hline A9 & 0.0 & 21.5 & 0 & Applicator & 700 & 100 & yes & 100 & yes & 100 & 100 \\
\hline A10 & 6.7 & 20.2 & 0 & Applicator & 700 & 100 & yes & 100 & yes & 100 & 100 \\
\hline A11 & 10.4 & 17.5 & 0 & Applicator & 700 & 100 & yes & 100 & yes & 100 & 100 \\
\hline $\mathrm{A} 12$ & 15.0 & 13.0 & 0 & Applicator & 700 & 100 & yes & 100 & yes & 100 & 100 \\
\hline $\mathrm{A} 13$ & 18.7 & 5.0 & 0 & Applicator & 700 & 100 & yes & 100 & yes & 100 & 100 \\
\hline A14 & 20.0 & 0.0 & 0 & Applicator & 700 & 100 & yes & 100 & yes & 100 & 100 \\
\hline A15 & 20.0 & -5.0 & 0 & Applicator & 700 & 100 & yes & 100 & yes & 100 & 100 \\
\hline A16 & 20.0 & -10.0 & 0 & Applicator & 700 & 100 & yes & 100 & yes & 100 & 100 \\
\hline A17 & 20.0 & -15.0 & 0 & Applicator & 700 & 100 & yes & 100 & yes & 100 & 100 \\
\hline $\mathrm{A} 18$ & 20.0 & -20.0 & 0 & Applicator & 700 & 100 & yes & 100 & yes & 100 & 100 \\
\hline
\end{tabular}

method as well as proposed method. The time needed to generate an acceptable treatment plan was measured using a stop watch. This time did not account for the importing CT scans as they were already in the system. Moreover, the time for pushing the treating plan to the treatment machine and printing the plan was also not included as they will be common regardless of the planning method chosen. The respective planning times were $9.3 \pm 0.2$ minutes vs. 4.9 \pm 0.3 minutes showing an advantage of $\sim 53 \%$. It is important that the proposed method also improves the consistency of dose points definition at the cylinder surface, as well as at $5 \mathrm{~mm}$ from it and hence the dose delivery accuracy from patient to patient.

\section{Discussion}

Vaginal cylinder HDR brachytherapy has been used with increasing frequency in the treatment of endometrial cancer, cervical cancer and cancer of the vagina. Despite significant technological advances in medical imaging, external beam radiation, treatment planning and delivery, few changes have occurred in planning and delivery of vaginal cylinder brachytherapy. Though multi-channel catheter based vaginal applicators have been suggested by some users, single catheter based applicators remain the most common applicator for these treatments [12]. In the clinic, the added cost of more sophisticated CT/MR based applicators which have a limited life-expectancy and may require frequent expensive replacements, the latest 3D brachytherapy planning systems, as well as the physics/dosimetry staff required to manage them, has made it difficult to transition to $3 \mathrm{D}$ brachytherapy, particularly in the absence of any proven or expected clinical benefit. Despite these obstacles, there is a desire to incorporate the available 3D information in modern planning [10]. Our proposed library-based technique provides an easy method that uses existing resources to migrate from 2D to 3D brachytherapy with minimal changes in the work flow and without requiring the purchase of additional equipment. Although additional time is needed to contour organs at risk and determine dosimetric indices (for example $\mathrm{D}_{2 \mathrm{cc}}, \mathrm{D}_{1 \mathrm{cc},}, \mathrm{D}_{0.1 \mathrm{cc}}$ ) as per GEC-ESTRO guidelines, the proposed method will significantly reduce 
Table 4. Standard treatment plan library with first 7 active dwell positions for $3.5 \mathrm{~cm}$ cylinder diameters for stainless steel vaginal cylinder applicator (Nucletron). Included are coordinates of the points on the applicator surface and at a distance of $5 \mathrm{~mm}$; also included is the location of the dwell position coincident with the origin of the treatment plan. It is important to know that in this table the dose values shown at cylinder surface and at $5 \mathrm{~mm}$ from it are for representative purposes only. Their actual values will change based on the dose-prescription, normalization as well as optimization selected by the user on the fly

\begin{tabular}{|c|c|c|c|c|c|c|c|c|c|c|c|}
\hline \multicolumn{12}{|c|}{ Origin $=4^{\text {th }}$ dwell position } \\
\hline \multicolumn{12}{|c|}{ Applicator pts - surface } \\
\hline Name & $x[\mathrm{~mm}]$ & $y[\mathrm{~mm}]$ & $z[\mathrm{~mm}]$ & $\begin{array}{l}\text { Coord. } \\
\text { system }\end{array}$ & $\begin{array}{c}\text { Act. dose } \\
\text { [cGy] }\end{array}$ & $\begin{array}{l}\text { Act. dose } \\
(\%)\end{array}$ & $\begin{array}{l}\text { Normali- } \\
\text { zation }\end{array}$ & $\begin{array}{c}\text { Norm. } \\
\text { dose }(\%)\end{array}$ & $\begin{array}{l}\text { Optimi- } \\
\text { zation }\end{array}$ & $\begin{array}{c}\text { Opt. rel. } \\
\text { dose }\end{array}$ & $\begin{array}{c}\text { Opt. } \\
\text { weight }\end{array}$ \\
\hline $\mathrm{A} 1$ & 0.0 & 21.5 & 0 & Applicator & 1000 & 100 & no & 100 & no & 100 & 100 \\
\hline $\mathrm{A} 2$ & 4.5 & 21.0 & 0 & Applicator & 1000 & 100 & no & 100 & no & 100 & 100 \\
\hline A3 & 7.8 & 20.0 & 0 & Applicator & 1000 & 100 & no & 100 & no & 100 & 100 \\
\hline A4 & 12.5 & 16.0 & 0 & Applicator & 1000 & 100 & no & 100 & no & 100 & 100 \\
\hline A5 & 16.3 & 10.0 & 0 & Applicator & 1000 & 100 & no & 100 & no & 100 & 100 \\
\hline A6 & 17.5 & 5.0 & 0 & Applicator & 1000 & 100 & no & 100 & no & 100 & 100 \\
\hline A7 & 17.5 & 0.0 & 0 & Applicator & 1000 & 100 & no & 100 & no & 100 & 100 \\
\hline$A 8$ & 17.5 & -5.0 & 0 & Applicator & 1000 & 100 & no & 100 & no & 100 & 100 \\
\hline A9 & 17.5 & -10.0 & 0 & Applicator & 1000 & 100 & no & 100 & no & 100 & 100 \\
\hline A10 & 17.5 & -15.0 & 0 & Applicator & 1000 & 100 & no & 100 & no & 100 & 100 \\
\hline \multicolumn{12}{|c|}{ Applicator pts $-5 \mathrm{~mm}$ from applicator surface } \\
\hline Name & $x[\mathrm{~mm}]$ & $y[\mathrm{~mm}]$ & $z[\mathrm{~mm}]$ & $\begin{array}{l}\text { Coord. } \\
\text { system }\end{array}$ & $\begin{array}{c}\text { Act. dose } \\
{[\mathrm{cGy}]}\end{array}$ & $\begin{array}{l}\text { Act. dose } \\
(\%)\end{array}$ & $\begin{array}{c}\text { Normali- } \\
\text { zation }\end{array}$ & $\begin{array}{c}\text { Norm. } \\
\text { dose }(\%)\end{array}$ & $\begin{array}{l}\text { Optimi- } \\
\text { zation }\end{array}$ & $\begin{array}{c}\text { Opt. rel. } \\
\text { dose }\end{array}$ & $\begin{array}{c}\text { Opt. } \\
\text { weight }\end{array}$ \\
\hline A11 & 0.0 & 26.5 & 0 & Applicator & 700 & 100 & yes & 100 & yes & 100 & 100 \\
\hline A12 & 6.5 & 25.5 & 0 & Applicator & 700 & 100 & yes & 100 & yes & 100 & 100 \\
\hline A13 & 10.5 & 23.5 & 0 & Applicator & 700 & 100 & yes & 100 & yes & 100 & 100 \\
\hline A14 & 16.0 & 18.5 & 0 & Applicator & 700 & 100 & yes & 100 & yes & 100 & 100 \\
\hline A15 & 19.0 & 15.0 & 0 & Applicator & 700 & 100 & yes & 100 & yes & 100 & 100 \\
\hline A16 & 22.0 & 10.0 & 0 & Applicator & 700 & 100 & yes & 100 & yes & 100 & 100 \\
\hline A17 & 22.5 & 5.0 & 0 & Applicator & 700 & 100 & yes & 100 & yes & 100 & 100 \\
\hline A18 & 22.5 & 0.0 & 0 & Applicator & 700 & 100 & yes & 100 & yes & 100 & 100 \\
\hline A19 & 22.5 & -5.0 & 0 & Applicator & 700 & 100 & yes & 100 & yes & 100 & 100 \\
\hline $\mathrm{A} 20$ & 22.5 & -10.0 & 0 & Applicator & 700 & 100 & yes & 100 & yes & 100 & 100 \\
\hline A21 & 22.5 & -15.0 & 0 & Applicator & 700 & 100 & yes & 100 & yes & 100 & 100 \\
\hline
\end{tabular}

the overall planning time to only slightly more than the time required for standard 2D planning. It is important to know that GEC-ESTRO indices need not be defined at the time of the treatment planning, but could be easily handled on retrospective basis as this is done for dose reporting purposes, not for modifying the treatment plans. This also may reduce treatment planning times and maintain patient through-put.

Another advantage of this method is that the process could be easily adapted for non-standard geometries that are patient and disease specific. Additional dose-points at surface or at $5 \mathrm{~mm}$ or at any other location could be easily added either as an extension of existing points using applicator coordinate system or even dropping them directly, using existing CT scans using patient coordinate system. This is due to the fact that the critical geometry part of defining points is on the round part towards the tip of the ap- plicator (i.e. the first ovoid in the applicator) which is well defined by this method.

\section{Conclusions}

According GEC-ESTRO Guidelines, the transition from 2D to 3D image based planning for vaginal cylinder HDR brachytherapy requires segmenting of various organs at risk as well as measurement of new dose volume parameters $\left(\mathrm{D}_{2 \mathrm{cc},}, \mathrm{D}_{1 \mathrm{cc},} \mathrm{D}_{0.1 \mathrm{cc}}\right)$ in addition to the standard reported 2D dose points for bladder, rectum etc. This increases planning time and effort in the absence of an expected benefit in clinical outcome. Despite this, it is important to continue supporting the transition to $3 \mathrm{D}$ planning, because the collection of volumetric data is an essential step in determining more accurate dose limits for organs at risk as well as for estimating the probability of treatment related toxicity. This 


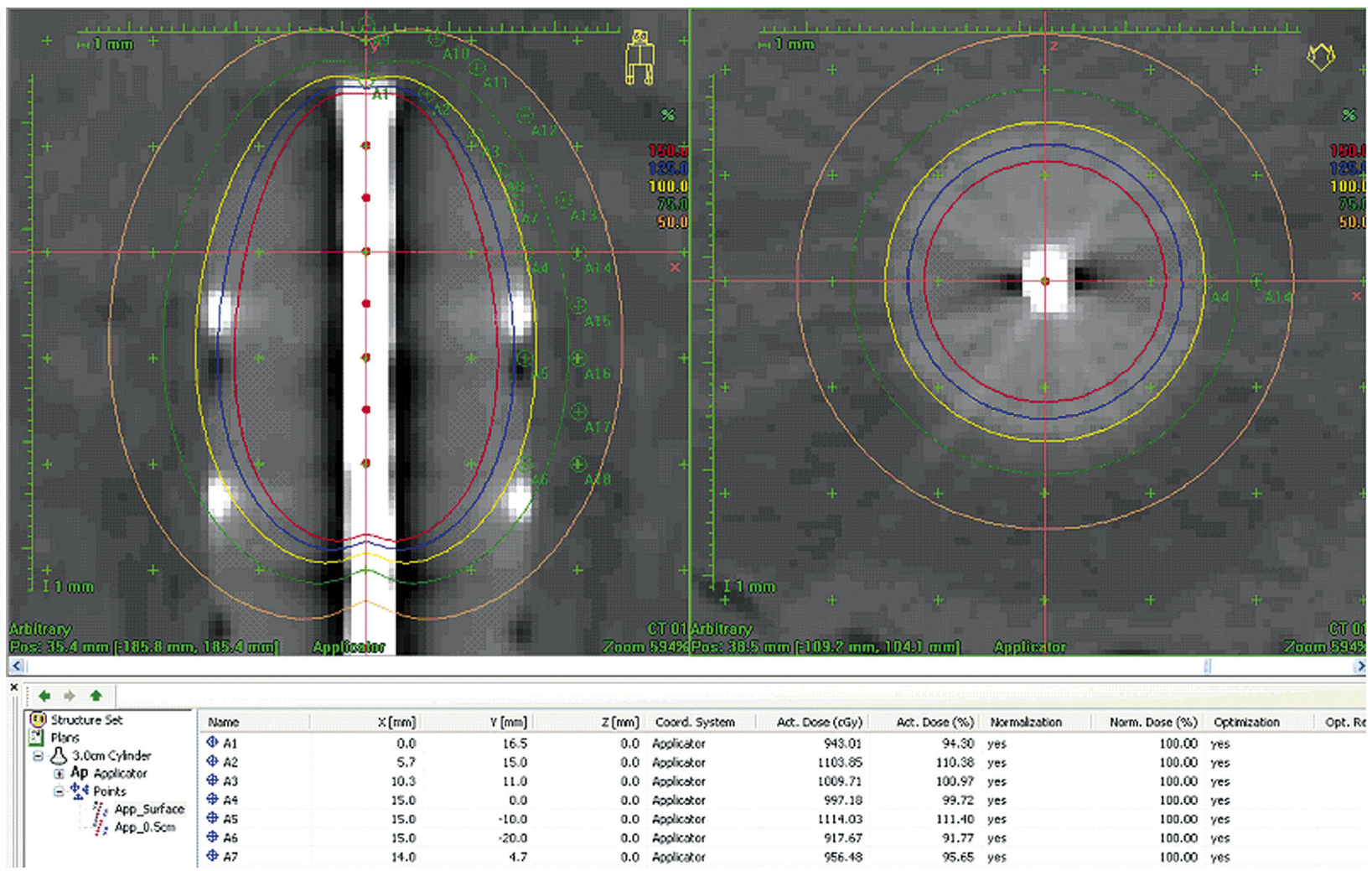

Fig. 1. A screen capture showing the dose-points overlaid on a patient CT scan. Also shown are the isodose curves and other relevant information

may allow for customization of plans to better suit patients in the future. This may even help in the comparison of multi-channel based applicators versus single catheter based applicators as well as the importance of angle of insertion (natural versus parallel to the treatment couch) of these devices in a patient's cavity in a scientific manner.

The proposed method involves creation of a standard plan library in Microsoft Excel along with simple windows operations of "copy" and "paste", could improve work flow efficiency, decreases overall planning time to approximately 5-10 minutes and reduces the chance for planning related errors. Though the method has been tested successfully on standard stainless steel vaginal applicator (Nucletron), it can be used with any other similar applicator as well and could be easily implemented in any Radiation Oncology department. The system is in use in our department since last 1.5 year and has been found to be very useful.

\section{Acknowledgement}

Authors would like to express their sincere thanks to Dr. Michael Kuettel, MD, PhD, MBA and Dr. Kilian Salerno-May, MD for many useful suggestions in the preparation of the manuscript.

\section{References}

1. Tan YI, Choo BA, Lee KM. 2D to 3D evaluation of organs at risk doses in intracavitary brachytherapy for cervical cancer. J Contemp Brachyther 2010; 2: 37-43.

2. Nout RA, van de Poll-Franse LV, Lybeert ML et al. Long-term outcome and quality of life of patients with endometrial car- cinoma treated with or without pelvic radiotherapy in the post operative radiation therapy in endometrial carcinoma 1 (PORTEC-1) Trial. J Clin Oncol 2011; 29: 1692-1700.

3. Nout RA, Smit VT, Putter H et al. Vaginal brachytherapy versus pelvic external beam radiotherapy for patients with endometrial cancer of high-intermediate risk (PORTEC-2): an open-label, non-inferiority, randomized trial. Lancet 2010; 375: 816-823.

4. Nout RA, Putter H, Jürgenliemk-Schulz IM et al. Quality of life after pelvic radiotherapy or vaginal brachytherapy for endometrial cancer: first results of the randomized PORTEC-2 trial. J Clin Oncol 2009; 27: 3547-3556.

5. Kushner DM, Fleming PA, Kennedy AW et al. High Dose Rate 192Ir Afterloading Brachytherapy for Cancer of the Vagina. Br J Radiology 2003; 76: 719-725.

6. McCloskey S, Tchabo NE, Malhotra HK et al. Adjuvant vaginal brachytherapy alone for high risk localized endometrial cancer as defined by the three major randomized trials of adjuvant pelvic radiation. Gynecol Oncol 2010; 116: 404-407.

7. Alektiar KM, Venkatraman E, Chi DS et al. Intravaginal brachytherapy alone for intermediate-risk endometrial cancer. Int I Radiat Oncol Biol Phys 2005; 62: 111-117.

8. Solhjem MC, Petersen IA, Haddock MG. Vaginal Brachytherapy alone is sufficient adjuvant treatment of surgical stage I endometrial cancer. Int J Radiat Oncol Biol Phys 2005; 62: 1379-1384.

9. Nag S, Brickson B, Prikh S, et al. The American Brachytherapy Society recommendations for high-dose-rate-brachytherapy for carcinoma of the endometrium. Int J Radiat Oncol Biol Phys 2000; 48: 779-790.

10. Potter R, Haie-Meder C, Limbergen EV et al. Recommendations from gynecological (GYN) GEC ESTRO working group (II): concepts and terms in 3D image-based treatment planning 
in cervix cancer brachytherapy-3D dose volume parameters and aspects of 3D image-based anatomy, radiation physics, radiobiology. Radiother Oncol 2006; 78: 67-77.

11. McMeekin S, Randall ME, Yashar C. A phase III trial of pelvic radiation therapy versus vaginal cuff brachytherapy followed by paclitaxel/carboplatin chemotherapy in patients with high risk, early stage endometrial carcinoma. GOG-0249 Protocol. October 9, 2009.

12. Demanes DJ, Rege S, Rodriguez RR et al. The use and advantages of a multichannel vaginal cylinder in high dose brachytherapy. Int J Radiat Oncol Biol Phys 1999; 44: 211-219. 